\title{
Linx
}

Revue des linguistes de l'université Paris X Nanterre

11 | 1999

Typologie des langues, universaux linguistiques

\section{Typologie des relatives et théorie de la relative}

\section{Alain Lemaréchal}

\section{OpenEdition}

\section{Journals}

Édition électronique

URL : http://journals.openedition.org/linx/888

DOI : $10.4000 /$ linx.888

ISSN : 2118-9692

\section{Éditeur}

Presses universitaires de Paris Nanterre

\section{Édition imprimée}

Date de publication : 1 décembre 1999

Pagination : 89-103

ISSN : 0246-8743

\section{Référence électronique}

Alain Lemaréchal, «Typologie des relatives et théorie de la relative », Linx [En ligne], 11 | 1999, mis en ligne le 29 juin 2012, consulté le 30 avril 2019. URL : http://journals.openedition.org/linx/888 ; DOI : 10.4000/linx.888

Ce document a été généré automatiquement le 30 avril 2019.

Département de Sciences du langage, Université Paris Ouest 


\title{
Typologie des relatives et théorie de la relative
}

\author{
Alain Lemaréchal
}

1 Comme on a parlé du «Problème de l'article et sa solution en ... », du «Problème de la préposition et sa solution en ... », on pourrait parler du «Problème de la relative et sa solution à travers la diversité des langues ». Ce genre de titre nous rappelle que toute approche translinguistique d'un problème, amène, qu'on le veuille ou non, à adopter un point de vue onomasiologique, mais, évidemment, il y a un risque : c'est qu'il n'y ait de «problème de l'article» que dans les langues qui en ont un et de "problème de la relative » que dans les langues qui en ont, c'est-à-dire qui ont tel ou tel type de structures particulières; bien sûr, les linguistes qui ont adopté cette façon de s'exprimer avaient autre chose en vue : il y a un problème au-delà de l'article, au-dessus de la diversité des langues, entre langues à vs sans article, celui qu'on pourrait appeler le problème de la détermination. Mais la première question à se poser, c'est : jusqu'où faut-il aller dans l'abstraction pour atteindre cet « au-dessus de la diversité »?

\section{Problèmes de délimitation et de généralisation}

Dans le cas de la relative, il faut sans nul doute monter très haut, ne serait-ce que parce que l'on construit les termes avec des prédicats (j'adopte résolument une position quinienne ${ }^{1}$ qui met les noms du côté des prédicats et non du côté des termes comme le fait Strawson ${ }^{2}$ ). Du coup, toute fonction prédicative au sens sémantico-logique du terme, peut contribuer à la construction d'une désignation à condition d'avoir une de ses places d'argument laissée ouverte : c'est l'exemple de Dik (1989, p. 55) :

\begin{tabular}{|l|l|}
\hline & le gros éléphant qui vit au zoo \\
\hline & $\mathrm{x}_{\mathrm{i}}$ : éléphant $\left(\mathrm{x}_{\mathrm{i}}\right): \operatorname{gros}\left(\mathrm{x}_{\mathrm{i}}\right): \operatorname{vivre}\left(\mathrm{x}_{\mathrm{i}} \mathrm{zoo}\right)$ \\
\hline
\end{tabular}




\begin{tabular}{|l|l|}
\hline càd & « Soit un $\mathrm{x}$, \\
\hline \hline & + dont on peut dire qu'il appartient à la classe, ouverte, des « éléphants », \\
\hline & + dont on peut dire qu'à l'intérieur de cette classe il est « gros » \\
\hline \hline & et qu'il « vit au zoo » \\
\hline
\end{tabular}

3 C'est parce que « vivre » a une de ses variables laissée ouverte que « vivre au zoo » peut déterminer "éléphant», mais on peut en dire autant pour "gros", et même pour " éléphant » : c'est parce que la variable est laissée ouverte que "gros » et «éléphant » peuvent aider à construire la référence d'un $x$. Mais les langues reflètent en fin de compte peu souvent dans leur structure syntaxique une formule aussi simple. Finalement, il n'y a guère que les langues dites « omniprédicatives » qui semblent au moins superficiellement se conformer à ce schéma ; ainsi, en nahuatl, on a :

\begin{tabular}{|l|l|}
\hline & cualli in tlācatl « l'homme est bon » \\
\hline vs & in tlācatl cualli « l'homme bon » \\
\hline \hline & cochi in pilli « l'enfant dort » \\
\hline & in pilli cochi (mais aussi in pilli in cochi) « l'enfant qui dort » \\
\hline
\end{tabular}

mais les langues omniprédicatives posent d'autres problèmes : si on prend en compte ce qui fournit les équivalents de nos relatives substantivales (du type défini «celui qui a téléphoné » ou du type générique « qui m'aime...»), ces langues étant omniprédicatives, on aura en fait la même structure pour un "celui qui a téléphoné », un "celui qui est rouge (le rouge) », et un « celui qui est homme (l'homme) »; si on étend encore l'usage de la notion de "relative» à ces cas, tout syntagme constituant un terme, y compris le syntagme nominal le plus simple ${ }^{4}$, sera à verser dans les relatives :

in cualli « le beau »

in cochi « celui qui dort »

in tlācatl «l'homme»

4 Ce n'est sans doute guère recommandable : il y aurait des subordonnées partout, des relatives partout; la notion se diluerait complètement. On ne devra toutefois pas oublier l'existence de ce type de situations. 


\section{Typologie et comparabilité}

5

Quant à la comparabilité (pour reprendre la problématique abordée par Gilbert Lazard), je soutiendrai qu'elle ne peut être assurée que par une atomisation maximale aussi bien de la forme que du sens :

1) une atomisation maximale du signifiant: le marquage des relations sémantiques, des structures syntaxiques, etc., est assuré par la «superposition» de marques de types divers ${ }^{5}$ :

- non seulement des marques segmentales, en l'occurrence : pronoms relatifs, marques diverses de relativisation, mais également prépositions, marques de diathèse, etc. ;

- mais aussi des marques intégratives (marquant l'intégration des segments à l'intérieur de segments englobants d'un niveau supérieur et signalant, entre autres, les limites des domaines de différents niveaux), en l'occurrence : l'enchâssement ;

- des marques séquentielles (ordre des mots), en l'occurrence : ordre déterminant + déterminé ou déterminé + déterminant ;

- des marques catégorielles, en l'occurrence : appartenance à une catégorie particulière (adjectifs, formes adjectivales ou nominales du verbe), valence et orientations des segments, en particulier de la forme verbale subordonnée, mais aussi des propositions subordonnées elles-mêmes (ces notions sont à étendre aux noms, propositions, etc. ${ }^{6}$ ) ;

2) une atomisation non moins radicale des signifiés, à savoir, dans le cas présent, des valeurs grammaticales, en atomes de signifié, c'est-à-dire, pour ne pas les nommer, en sèmes, représentables idéalement sous forme de $f(x), f(x, y)$ et de combinaisons de ces $f(x), f$ $(x, y)$; ce qui ne préjuge pas du niveau d'association entre forme et sens : certaines valeurs étant associées aux atomes de signifiant, d'autres l'étant au contraire aux segments construits les plus complexes.

Pour un phénomène comme la subordination, on devra donc se poser la question : que marque au juste chacune des marques associées à la subordination (enchâssement, morphèmes dits de "subordination », formes verbales particulières, etc.) ?

\section{Quelques types de relatives : ouvrir ou ne pas ouvrir de places d'argument?}

9 Je passerai en revue quelques types de relatives (je reprends ici les étiquettes de Givón ${ }^{7}$ ) : 1) la « Gap Strategy » avec le japonais, 2) les « relatives à pronom relatif » avec le français et l'anglais et 3) une des « Verb coding Strategies » avec le tagalog; je me limiterai à des types présentant des relatives (ou équivalents de relatives) enchâssées.

Je commencerai par les relatives du japonais illustrant la « Gap Strategy » de Givón, parce qu'on pourrait s'attendre à ce que des relatives de ce type reflètent directement la formule sémantico-logique de Dik.

11 En japonais, une proposition (qui pourrait constituer une indépendante dans le cas où le sujet est marqué par $g a^{8}$, à ceci près que l'argument coréférentiel de l'antécédent ne peut y être instancié) est enchâssée (marque intégrative) devant le nom à déterminer (marque séquentielle : ordre normal déterminant + déterminé dans cette langue), sans pronom relatif, ni une quelconque marque segmentale de relativisation ou de subordination: 


\begin{tabular}{|l|l|l|l|}
\hline otoko-ga & onna -ni & tegami-o & kaita \\
\hline \hline homme MSuj & femme MDat & lettre MAcc & écrire \\
\hline «the man sent a letter to the woman »(Givón, p. 658 $)$ \\
\hline
\end{tabular}

\begin{tabular}{|l|l|l|l|l|l|}
\hline$>$ & $\varnothing$ & onna $-n i$ & tegami-o & kaita & otoko \\
\hline & SUJET & femme MDat & lettre MAcc & écrire & homme \\
\hline \hline & «the man who sent a letter to the woman...» \\
\hline
\end{tabular}

\begin{tabular}{|l|l|l|l|l|l|}
\hline$>$ & otoko-ga & onna $-n i$ & $\emptyset$ & kaita & tegami... \\
\hline \hline & homme MSuj & femme MDat & OBJET & écrire & lettre \\
\hline & «the letter that the man sent to the woman...» \\
\hline
\end{tabular}

\begin{tabular}{|l|l|l|l|l|l|}
\hline$>$ & otoko-ga & $\varnothing$ & tegami-o & kaita & onna... \\
\hline & homme MSuj & DATIF & lettre MAcc & écrire & femme \\
\hline & \multicolumn{3}{|l}{ «the woman to whom the man sent a letter...» } \\
\hline
\end{tabular}

12 Un des problèmes que pose cette «gap strategy » est d'expliquer comment on récupère le rôle sémantique de l'antécédent par rapport à la relative.

13 Généralement, on considère (c'est le cas de Givón) qu'on y parvient à l'aide d'une inférence par soustraction : on connait la valence du verbe de la relative; dans l'exemple : kaita « écrire » peut être considéré comme un verbe triactanciel, c'est-à-dire exprimant une fonction prédicative (au sens sémantico-logique du terme) à trois places d'argument ; deux de ces arguments étant instanciés et leurs fonction et rôle sémantique étant explicitement indiqués par les marques de cas postposées à chacun d'eux, on inférera par soustraction le rôle de l'« argument absent». On voit immédiatement que cela ne peut marcher pour les circonstants, les circonstants étant par définition étrangers à la valence du verbe :

\begin{tabular}{|l|l|l|}
\hline musume-ga & kekkonsi -ta & tosi ... \\
\hline fille MSuj & se-marier MPass & année \\
\hline \hline « l'année où ma fille s'est mariée .... \\
\hline
\end{tabular}

14 Dans musume-ga kekkonsita, «(ma) fille s'est mariée », il est tout à fait artificiel de poser un CircTps $\varnothing$ absent, il s'agit d'une proposition complète sans place vide. Ce qui suggère 
l'interprétation «l'année où ma fille s'est mariée » est évidemment le sens de l'antécédent lui-même; c'est seulement après qu'on restituera un CircTps $\emptyset$.

Cela nous apprend trois choses absolument essentielles pour une théorie de la relative : 1) il n'y a pas nécessairement de place vide dans la relative, 2) les inférences partent de la tête, 3) un nom peut être simplement déterminé par une proposition, la relation entre les deux restant non spécifiée, ce que j'appelle « relation minimale », un type de relation que je crois fondamentale dans les langues ${ }^{10}$; on doit gloser : «l'année déterminée par le fait que $\mathrm{P} \gg$ :

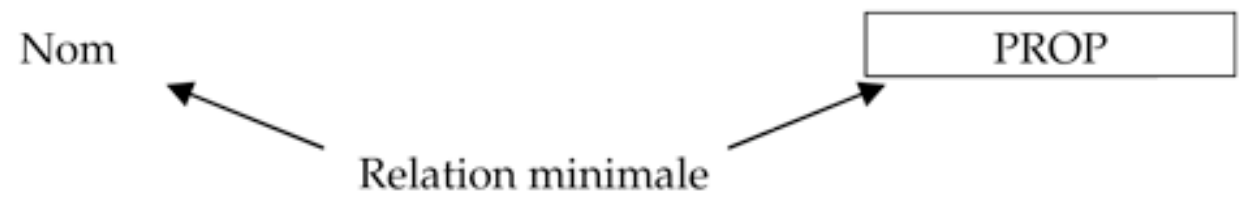

Même dans le cas de la relativisation de rôles actanciels, on s'aperçoit qu'il en va de même en japonais : pour que l'inférence par soustraction soit automatique, il faudrait qu'en japonais tous les arguments soient obligatoirement instanciés, ce qui n'est pas le cas dès que le participant est évident du fait du contexte ou de la situation ou, inversement, qu'il est laissé non spécifié :

\begin{tabular}{|l|l|l|l|l|}
\hline$\varnothing$ & $\varnothing$ & tegami-o & kaita & otoko \\
\hline \hline SUJET & DATIF & lettre MAcc & écrire & homme \\
\hline «l'homme qui a écrit la lettre ...» & & \\
\hline \hline «l'homme à qui a été écrite la lettre $(=$ « à qui on $/ \mathrm{je} / \mathrm{tu} / \mathrm{il} \ldots »)$ \\
\hline
\end{tabular}

Là encore, l'inférence part de la tête, la formule argumentale du verbe n'intervient que dans une étape ultérieure et la sélection finale de la bonne interprétation nécessite une information plus large au delà du domaine étroit du syntagme. Il existe même un certain nombre de relations qui ne sont pas facilement paraphrasables ${ }^{11}$ :

\begin{tabular}{|l|l|l|l|}
\hline Nakama & $-o$ & uragit -ta & batu... \\
\hline \hline camarade & Mobj & trahir MTps & punition \\
\hline « la punition (qui découle du fait) qu'il a trahi ses camarades ...» & \\
\hline
\end{tabular}

18 Il n'est pas étonnant qu'une frontière passe entre relativisation d'un argument du prédicat subordonné et relativisation de participants étrangers à cette valence. L'existence de deux ou trois stratégies distinctes selon la fonction ou le rôle sémantique à relativiser est un phénomène fréquent ; entre sujet et objet vs circonstant, entre sujet vs objet vs circonstant et tiers actant. C'est le cas en ponape (Givón, p. 676 sqq.). Le sujet et l'objet sont relativisés par simple enchâssement (me est facultatif) avec postposition à l'ensemble antécédent + relative suivi de l'article -o ou des démonstratifs : 


\begin{tabular}{|l|l|l|l|l|}
\hline & lii & kilang & ool & «the woman saw the man » \\
\hline & woman & see & man & \\
\hline
\end{tabular}

\begin{tabular}{|l|l|l|l|l|l|}
\hline$>$ & lii & me & kilang & ool-o & «the woman who saw the man... » \\
\hline & woman & Rel see & man & Dém & \\
\hline
\end{tabular}

\begin{tabular}{|l|l|l|l|l|l|}
\hline$>$ & ool & me & lii & kilang-o & «the man that the woman saw... » \\
\hline & man & Rel & woman & see Dém & \\
\hline
\end{tabular}

La relativisation de l'instrument passe par l'objectivisation de l'instrument au moyen d'un relateur intraverbal :

\begin{tabular}{|l|l|l|l|l|l|}
\hline & ool-o & soroo & utuk-o & ki-naip & $(k i=$ préposition $)$ \\
\hline & man-Déf & cut & meat-Déf & with-knife & \\
\hline \hline & & "the man cut the meat with the knife » \\
\hline
\end{tabular}

\begin{tabular}{|l|l|l|l|l|l|}
\hline$>$ & ool-o & soroo- $k i$ & naip & $u t u k$ & $(k i=$ relateur intraverbal $)$ \\
\hline \hline & man-Déf & cut-Instr & knife & meat & \\
\hline \hline & \multicolumn{2}{|l}{ «the man used the knife to cut meat » } \\
\hline
\end{tabular}

\begin{tabular}{|l|l|l|l|l|l|}
\hline$>$ & naip-o & me & ool-o & soroo-ki & utuk-o \\
\hline & knife-Déf & Rel & man-Déf & cut-Instr & meat-Dém \\
\hline & « the knife with which the man cut the meat » \\
\hline
\end{tabular}

\begin{tabular}{|l|l|l|l|l|l|}
\hline vs & *naip-o & me & ool-o & soroo & utuk-o \\
\hline & knife-Déf & Rel & man-Déf & cut & meat-Dém \\
\hline
\end{tabular}

La relativisation du lieu passe par l'insertion d'un adverbe de lieu résomptif :

\begin{tabular}{|l|l|l|l|l|l|}
\hline deke & $(m e)$ & Soulik & ipwidi & iee & - -o \\
\hline \hline & island & S. & was-born & there & Dém \\
\hline
\end{tabular}


"... the island where Soulik was born» (japonais, turc), ou bien il faut ajouter des marques segmentales internes à la construction relative qui ouvrent une nouvelle place d'argument. Comme nous venons de le voir, plusieurs solutions sont possibles ; j'en étudierai ici plus particulièrement deux : ou bien une marque de diathèse du verbe subordonné (langues des Philippines, langues bantoues), ou bien une préposition (français, anglais, mais aussi berbère, langue sans pronom relatif), permet d'ouvrir une place d'argument en spécifiant le rôle sémantique de l'antécédent ; illustrons d'abord ce dernier cas :

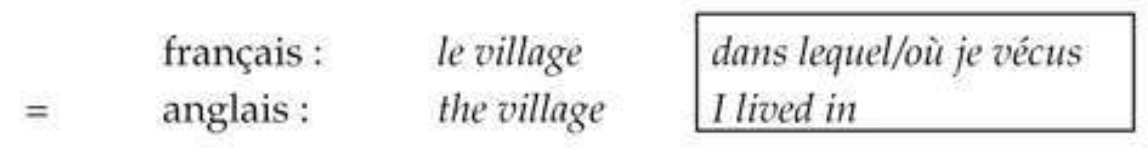

$$
\begin{aligned}
& \text { = segments orientés vers le lieu }
\end{aligned}
$$

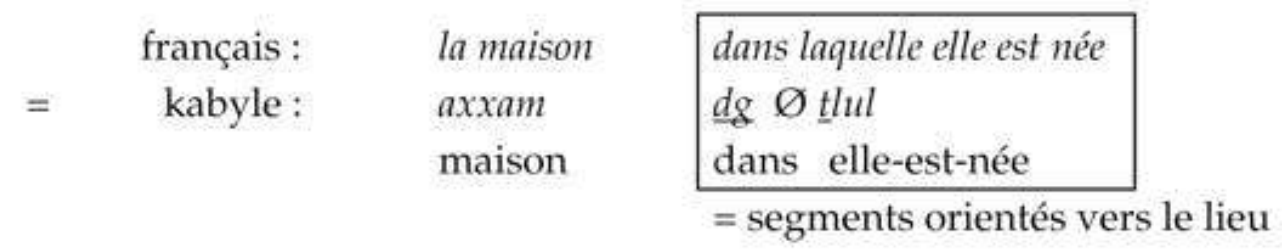

24 Le groupe préposition + pronom relatif (français ou anglais), ou la préposition sans régime (anglais ou berbère) antéposée ou postposée à la relative, apparait comme une marque spécifiant la relation entre l'antécédent et la relative, là où le japonais laisse cette relation non spécifiée :

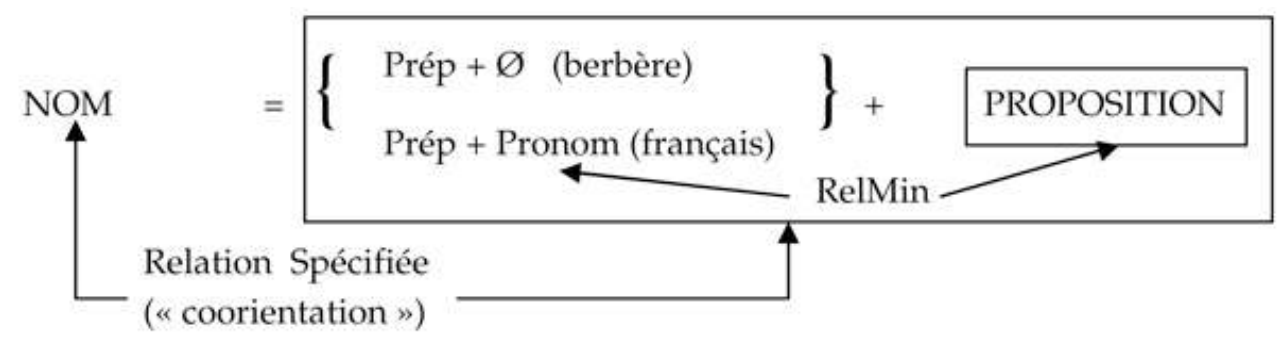

Le groupe préposition + relatif ou la préposition sans régime fonctionne comme une marque qui spécifie la relation entre le nom à déterminer antécédent et la proposition, c'est-à-dire comme une sorte de marque de voix enchâssée au niveau de la proposition toute entière, et non plus au niveau d'une forme verbale comme c'est le cas, par exemple, dans les relatives du tagalog.

En tagalog, l'accès à la relativisation est extrêmement limité en termes de fonction, puisque seul le sujet a accès à la relativisation (il n'y a, si l'on veut, que des relatives par 
QUI); pour cette langue, le point de rupture dans la hiérarchie d'accessibilité ${ }^{12}$ des fonctions à la relativisation se situe donc très haut :

SUJ $\|>$ OBJ1 > OBJ2 > OBJé > CIRC

27 L'accès est beaucoup plus large en termes de rôle sémantique :

agent $>$ patient $>$ dest ${ }^{\text {aire }}>$ bénéf aire $>$ instr $>$ lieu $\|>$ temps

28 Les voix verbales multiples, caractéristiques des langues des Philippines compensent le fait qu'il n'y ait que des relatives par QUI. Le tagalog possède des voix (ou focus ${ }^{13}$ ) permettant de subjectiviser (promouvoir en sujet) presque tous les rôles sémantiques : l'agent, le patient, le destinataire, mais aussi l'instrument, le bénéficiaire, la cause matérielle, le causateur, le lieu simple cadre d'un procès; je n'en illustrerai ici que quelques unes :

b-um-ili n-ang libro ang doktor $\quad($ Actif $=$ marque $-u m$ - $)$

« le médecin a acheté un livre »

\begin{tabular}{|l|l|l|}
\hline b-in-ili n-ang doktor & ang libro & (Passif = marque $\varnothing ;$ \\
\hline \hline « le livre a été acheté par un/le médecin » & -in-, m. d'accompli) \\
\hline
\end{tabular}

\begin{tabular}{|l|l|l|}
\hline s-in-ulat-an ko n-ang Y & ang doktor & (Voix destinative = marque -an) \\
\hline «j'ai écrit Y au médecin » \\
\hline (lit. « le médecin s'est vu écrire par moi... ») \\
\hline
\end{tabular}

\begin{tabular}{|l|l|}
\hline s-in-ulat-an ko $n$-ang $Y$ & (id. actant promu = actant local) \\
\hline «j'ai écrit Y sur le papier » \\
\hline (lit. « le papier est sur quoi j'ai écrit Y ») \\
\hline
\end{tabular}

\begin{tabular}{|l|l|}
\hline i-p-in-am-bili ko n-ang Y ang pera & (Voix instrumentive = marque i-paN-) \\
\hline «j'ai acheté Y avec l'argent » \\
\hline (lit. « l'argent est avec quoi j'ai acheté Y «)
\end{tabular}

\begin{tabular}{|l|l|}
\hline -in-ag-sulat-an ko ... ang desk & (Voix locative = marque pag- -an) \\
\hline$?$ « j'ai écrit ... sur le bureau $»^{14}$ \\
\hline \hline (lit. « le bureau est où j'ai écrit $Y$ «) \\
\hline
\end{tabular}


La relativisation consiste en l'enchâssement de la forme verbale à la voix correspondante, moyennant la marque de relativisation $n a(-n g$ après voyelle) :

\begin{tabular}{|l|l|l|l|l|}
\hline$>$ & ang doktor & $n a$ & b-um-ili ... & « le médecin qui a acheté ... » \\
\hline$>$ & ang libro & $-n g$ & b-in-ili ... & « le livre que le médecin a acheté » \\
\hline$>$ & ang pera & $-n g$ & i-p-in-am-bili ... & « l'argent avec lequel ... » \\
\hline$>$ & ang doktor & $n a$ & s-in-ulat-an ... & « le médecin auquel ... » \\
\hline$>$ & ang papel & $n a$ & $s$-in-ulat-an ... & « le papier sur lequel ... » \\
\hline$>$ & ang desk & $n a$ & $p$-in-ag-sulat-an & « le bureau sur lequel ... » \\
\hline
\end{tabular}

On constate que, malgré la différence de structure interne entre tagalog et français (voix verbales vs préposition), le résultat est le même: une spécification extrême des rôles sémantiques :

\begin{tabular}{|c|c|c|}
\hline \multirow[t]{2}{*}{ tagalog: } & $\begin{array}{l}\text { le médecin } \\
\text { ang doktor }\end{array}$ & 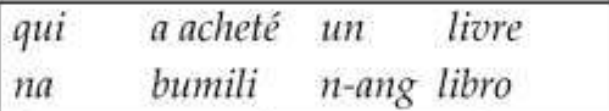 \\
\hline & & $\begin{array}{l}\text { Segments en fonction épithétique } \\
\text { orientés vers l'agent }\end{array}$ \\
\hline français : & le livre & $\begin{array}{l}\text { que le médecin a acheté } \\
\text { acheté par le médecin }\end{array}$ \\
\hline $\begin{array}{l}\text { tagalog: } \\
\text { anglais: }\end{array}$ & $\begin{array}{l}\text { ang libro } \\
\text { the book }\end{array}$ & $\begin{array}{l}\text {-ng b-in-ili n-ang doktor } \\
\text { the doctor bought }\end{array}$ \\
\hline & & $\begin{array}{l}\text { Segments en fonction épithétique } \\
\text { orientés vers le patient }\end{array}$ \\
\hline \multirow[t]{2}{*}{$\begin{array}{l}\text { français : } \\
\text { tagalog: }\end{array}$} & $\begin{array}{l}\text { l'argent } \\
\text { ang pera }\end{array}$ & $\begin{array}{ll}\text { avec lequel a été acheté } & \text { le livre } \\
-n g \text { i-p-in-ambili } & n \text {-ang libro }\end{array}$ \\
\hline & & $\begin{array}{l}\text { Segments en fonction épithétique } \\
\text { orientés vers l'instrument }\end{array}$ \\
\hline
\end{tabular}

Ce qui permet aux structures relatives des deux langues de s'insérer là où elles s'insèrent, c'est une caractéristique globale du segment, à savoir son orientation précise vers tel ou tel participant. Il faut étendre les notions de valence et d'orientation bien au-delà du verbe, aux noms, mais également aux propositions ${ }^{15}$. De même qu'une forme verbale marquée en diathèse et en voix est orientée vers un premier actant et spécifie le rôle de cet actant par rapport au procès exprimé par la base verbale (à l'actif, orientation vers l'agent, au passif vers le patient, etc. ${ }^{16}$, de même une proposition relative spécifie le rôle sémantique de l'antécédent par rapport à la relative. 
le que soit la structure interne du segment enchâssé, ce qui lui permet de s'intégrer dans le syntagme nominal comme déterminant, c'est son orientation, que celle-ci soit assurée par une préposition + relatif, une préposition sans régime, ou par la voix du verbe: le segment pris dans son entier fonctionne comme une "boîte noire $»^{17}$, et l'orientation est une caractéristique globale de cette boîte noire :
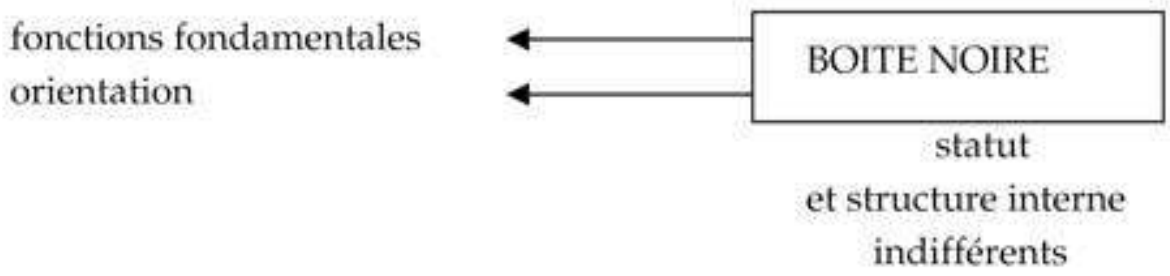

Si l'on entre dans la boîte noire, on s'aperçoit qu'en fin de compte la différence entre français, anglais, berbère et tagalog est une question de niveau d'intégration de la marque d'orientation : intégration à la proposition enchâssée, en français, anglais, berbère, vs intégration à la forme verbale en tagalog.

Cette différence d'intégration passe, d'une façon bien connue, entre intégration aux limites du domaine, c'est-à-dire avant ou après (sans compter la "position de Wackernagel »), et intégration à la tête du domaine, c'est-à-dire le prédicat subordonné, changement d'intégration qui ne fait d'ailleurs que reporter sur le domaine de tête le choix entre position avant vs position après :

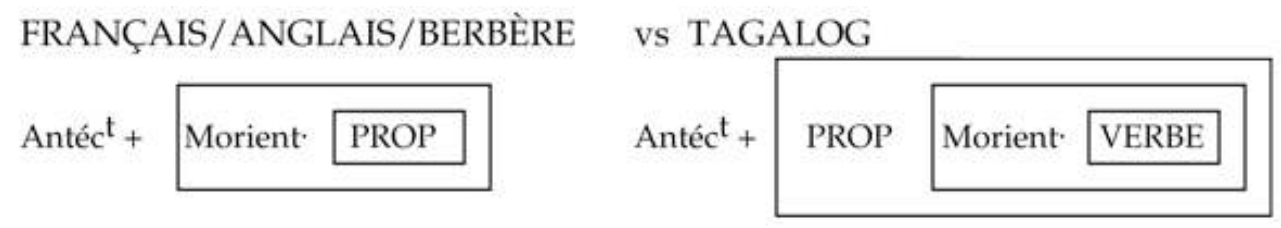

On ne sera pas surpris d'apprendre qu'une partie des marques de voix du tagalog provient sans doute de prépositions, -an, (et également de marques de nom verbal, c'està-dire de " nom de proposition ", comparable au nom verbal en -dIk du turc et fonctionnant comme équivalent de complétive : entre autres, la marque pag-) et il est vraisemblable qu'une partie au moins des voix multiples se soit développée dans des constructions à fortes contraintes, comme, précisément, la relativisation, mais aussi la focalisation, la thématisation, etc.

J'ai montré ailleurs ${ }^{18}$ qu'il peut y avoir passage de préposition à relateur intraverbal, puis, à marque d'objectivisation (c'est-à-dire de promotion en objet), et, de là, à marque de subjectivisation, c'est-à-dire à marque de voix, ce dernier passage de marques d'objectivisation à marques de subjectivisation s'opérant à l'occasion d'un passage de construction accusative à construction ergative :

$$
\mathrm{V}(\mathrm{x}, \ldots . .)+\text { Prép }(\mathrm{X}, \mathrm{z})>\mathrm{V}+\text { RelIntraV }>\mathrm{V}+\mathrm{MObj} \cdot(\mathrm{x}, \ldots, \mathrm{z})>\text { Msubj. }
$$

Il s'agit de changements de diathèse et d'orientation de la forme verbale ${ }^{19}$.

38 Je proposerai la généralisation suivante. Le changement du rôle de la préposition, ou de tout autre relateur, quand de marque de complément elle devient marque d'orientation est analogue à un changement de voix.

Si on représente toute relation marquée par une préposition, postposition, marque de cas, etc., sous la forme d'un : 
$\mathrm{R}_{\text {Prép }}(\mathrm{X}, \mathrm{z})$

où $\mathrm{X}$ représente l'incidence de la préposition ${ }^{20}$,

et $\mathrm{z}$ le terme régime,

On peut dire que quand le groupe Préposition + Pronom ou la Préposition seule fonctionne comme marque d'orientation préposée ou suffixée à une construction relative, il y a un changement de hiérarchie entre $X$ et $z$, on passe d'une orientation primaire vers $X$ à une orientation primaire vers $z^{21}$ : une différence de voix, en fin de compte.

\section{Conclusion. Spécification vs non spécification : contraintes et émergence de formes}

41 Le type de spécification progressive de la relation sur un fond de relation non spécifiée que nous avons observé quand on passe du japonais au français comme au berbère ou au tagalog entre parfaitement dans un modèle de théorie linguistique où l'on considère les structures linguistiques comme "émergence de forme» et non comme manifestation d'une grammaire formalisée sous-jacente.

En fait, plusieurs facteurs poussent d'un côté à la spécification, de l'autre à la nonspécification:

43 1) d'abord un couple de pression antagoniste : d'une part, pouvoir déterminer un nom à l'aide de la plus grande diversité de $\mathrm{P}$; d'autre part, désambiguïser les relations possibles entre nom et $\mathrm{P}$ (je veux parler ici de la désambiguïsation du rôle de l'antécédent par rapport à la relative, et non d'une autre désambiguïsation qui concerne elle l'incidence de la relative : quel nom est déterminé par tel $\mathrm{P}$ ? Les stratégies de désambiguïsation dans ce deuxième domaine reposent entre autres sur un ordre syntaxique strict ou sur des phénomènes d'anaphore à courte portée, je n'en parlerai pas) ;

2) une pression qui vient des cas où $\mathrm{P}$ ne sert plus seulement à déterminer un nom, mais sert à construire directement un terme : c'est le cas des relatives substantivales définies, du type celui qui a téléphoné, ou génériques du type qui m'aime me suive. Dans le premier type, on trouvera souvent articles ou démonstratifs, sources possibles de marques de relative constituées par d'anciens déterminants; dans le deuxième type, on trouvera souvent des noms supports antécédents, fonctionnant en fait comme des classificateurs, du genre le type qui, la chose/le truc qui, etc., autre source possible de marques de relative; c'est ce qu'illustre le ponape ${ }^{22}$, où me est un ancien nom signifiant « chose », fournissant d'abord un antécédent vide, et devenu simple marque (facultative), démarcative, de relativisation :

\begin{tabular}{|l|l|l|l|l|}
\hline ohl & - o & pirap & & «l'homme a volé » \\
\hline homme & Art/Dém & voler & & \\
\hline & & & & \\
\hline ohl (me) & pirap -o & & & «l'homme, celui qui a volé » \\
\hline & & & \ & «l'homme qui a volé » \\
\hline
\end{tabular}


3) mais il faut prendre encore en compte une autre source de pression que sont les effets de normalisation des paradigmes (y compris paradigmes de structures): le marquage d'un type particulier de relative devenant au gré de changements d'ordre des mots, etc., le marquage de toutes les relatives: tout le problème est de dégager au gré de quels remaniements dans les structures et dans le jeu des différentes marques superposées ont pu se produire de tels changements. J'en donne rapidement quelques exemples :

- il est possible que le relatif d'origine interrogatif-indéfini se soit étendu des relatives génériques à toutes les relatives via les cataphoriques ;

- le marquage des relatives substantivales peut, par passage de construction marquée à construction non marquée, s'étendre aux relatives épithétiques, via les relatives appositives (substantivales > nominales), du type le médecin, celui qui est venu hier,... ; cf. l'exemple du ponape :

\begin{tabular}{|l|l|l||l|l|}
\hline ohl & & pirap -o & & «l'homme qui a volé » \\
\hline me & & pirap -o & & " celui qui a volé » \\
\hline ohl & me & pirap -o & & «l'homme, celui qui a volé »³ \\
\hline & & & " & «l'homme qui a volé » \\
\hline
\end{tabular}

- le marquage des relatives propres aux niveaux de Dik ${ }^{24}$ supérieurs peut s'étendre aux niveaux inférieurs : le type de marquage nécessaire pour spécifier le rôle sémantique des circonstants de différents niveaux s'étendant alors au marquage de la relativisation des arguments.

\section{BIBLIOGRAPHIE}

DIK, S. C. (1989) The Theory of Functional Grammar, Part I : The Structure of the Clause, Dordrecht, Foris Publications.

GIVÓN, T. (1984-1989) Syntax. A functional-typological Introduction, I-II, Amsterdam, John Benjamins. LEMARÉCHAL, A . (1983) Sur la prétendue homonymie des marques de fonction : la superposition des marques, BSLP, 78/1, p. 53-76.

LEMARÉCHAL, A. (1989) Les parties du discours. Sémantique et syntaxe, Paris, PUF.

LEMARÉCHAL, A. (1997) Zéro(s), Paris, PUF.

LEMARÉCHAL, A (1998) Etudes de morphologie en $f(x)$, Louvain-Paris, Peeters.

QUINE, W. V. (1960) Word and Object (trad. fr. Le mot et la chose, Flammarion).

STRAWSON, P.F. (1959) Individuals, London, Methuen (trad. fr. Les individus, Editions du Seuil, 1973). 


\section{NOTES}

1. Quine 1960 (trad.fr., 1978), Le mot et la chose, p. 256 sqq.

2. Strawson 1959 (trad. fr., 1973), Les individus, en particulier, p. 147 sqq., 172 sqq., 220 sqq.

3. En anglais dans l'original : the big elephant that lives in the zoo. L'analyse précise de Dik est la suivante :

$$
\begin{aligned}
& \text { the big elephant that lives in the zoo }
\end{aligned}
$$

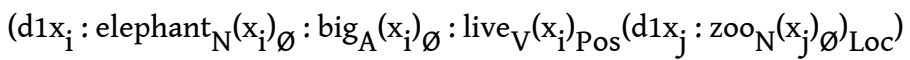

on voit qu'y figurent 1) l'indication des parties du discours (sans que soit problématisée la question de leur distribution et de leur définition), ici : N, A, et $\mathrm{V}, 2$ ) l'indication des rôles sémantiques (dont la catégorisation est présentée p. $100 \mathrm{sqq}$ ), ici : $\varnothing$ («Zero : entity primarily involved in a State», y compris l'argument des noms et des adjectifs), Pos («Positioner : the entity controlling a position ») et Loc ("Location : the place where something is located»), 3) l'indication de la détermination et de la quantification à travers une symbolisation qui évoque d'une façon peut-être quelque peu fallacieuse les quantificateurs logiques, ici : d1 «défini + singulier ».

4. Dans ce type de langues, les apories attachées au concept mal formé de syntagme nominal sont particulièrement visibles : appellera-t-on SN les syntagmes en in même ceux en in + Verbe, à l'exclusion des syntagmes en $\varnothing+$ Nom ? On voit que ce qui est essentiel, c'est l'opposition entre syntagmes avec in (fonctions actancielles, commutation avec les démonstratifs), ce que j'appelle des « syntagmes substantivaux », et sans in (fonction prédicative); cf. Lemaréchal 1989, chap. 1.

5. Cf. Lemaréchal 1983.

6. Cf. Lemaréchal 1989, chap. 7 à 9.

7. Givón, 1989, p. 650-679.

8. La marque de sujet ga peut commuter avec la marque de génitif no dans une subordonnée, et avec la marque de thématisation wa dans une principale (cf. Lemaréchal 1997, p. 145 sqq., pour une interprétation de ces faits).

9. Curieusement, Givón traduit kaita « écrire » par send « envoyer ».

10. Lemaréchal 1997, p. 102 sqq.

11. Je ne perds pas de vue que ce dernier type de relatives où aucune relation précise entre antécédent et subordonnée n'est restituable est exceptionnel (en japonais et en coréen seulement, semble-t-il), mais ce qui est exceptionnel, c'est que cette relation ne soit pas paraphrasable facilement; il est, en revanche, très courant que le rôle sémantique de l'antécédent demeure non spécifié et ne soit récupérable que par des inférences fondées en dernier ressort sur la vraisemblance (et puisse donner lieu à des ambiguïtés) : c'est le cas de tous les équivalents de relatives autres que par QUI en turc, autres que par QUI et QUE en malgache, par exemple.

12. Sur les notions de hiérarchie, de point de rupture, pour une présentation synthétique pratique, voir Dik 1989, p. 27-31.

13. Ce n'est pas le lieu de discuter de ce problème de terminologie. Quant à moi, je ne vois aucune raison de ne pas considérer un changement de forme verbale solidaire d'un changement de l'étiquetage casuel des actants comme un phénomène de voix, d'autant plus que l'actant sujet (prétendument « topic ») a certaines caractéristiques typiques des sujets.

14. La voix locative n'apparaît guère en tagalog que dans la relativisation, la focalisation, la thématisation.

15. Pour plus de détail, cf. Lemaréchal 1989 chap. 7 à 9, 1997 p. 136 sqq. 
16. On parlera d'« orientations secondaires » pour les participants privilégiés autres que le sujet (c'est-à-dire l'objet), ou simplement contrôlés par la forme verbale ; cf. Lemaréchal 1989, chap. $10-12$.

17. Cf. Lemaréchal 1997, p. 114 sqq.

18. Lemaréchal 1997, p. 200-211.

19. Portant d'abord sur l'orientation secondaire (objectivisation), puis primaire (subjectivisation).

20. Le nom dans le cas d'un syntagme prépositionnel complément de nom, le verbe dans le cas d'un syntagme prépositionnel complément de verbe, une prédication plus ou moins étendue dans le cas de syntagmes prépositionnels compléments circonstanciels, et même, la proposition dans le cas de syntagmes prépositionnels fonctionnant comme adverbe de phrase, et enfin une énonciation dans le cas de syntagmes prépositionnels fonctionnant comme adverbe d'énonciation; on retrouve les différents niveaux de prédication de Dik (1989, chap. 3), cf. Lemaréchal 1999, p. 145-147.

21. On peut noter :

$$
\mathrm{R}_{\text {Orient }}(\mathrm{z}, \mathrm{R}[\mathrm{X}, \mathrm{z}])
$$

22. Langue austronésienne parlée dans l'atoll du même nom ; cf. Rehg 1981.

23. Rehg fait, à tort selon moi, l'analyse inverse : il déduit la présence (facultative) de me dans les relatives de sa présence dans la focalisation. C'est une erreur : dans un grand nombre de langues de la famille, sinon dans toutes, la focalisation fait intervenir une proposition équative où le terme focalisé devient le prédicat et le reste de la proposition (y compris le verbe) le sujet, à l'aide d'une nominalisation, assurée par l'article substantivant dans les langues qui en ont un (par exemple en tagalog: doktor ang bumili nang libro « c'est un médecin qui a acheté le livre » ou, avec une proposition équative, ang doktor ang bumili nang libro «c'est le médecin qui a acheté le livre), ou par un antécédent vide («le truc», «le type », etc.) comme en ponape ohl-o me pirap " c'est l'homme qui a volé ». Quant à supposer qu'il y a toujours une focalisation sous-jacente à la relativisation, autant il est raisonnable d'en poser pour les interrogations partielles, autant ce n'est pas sans poser de problème à propos des relatives (cf. Lemaréchal 1997, p. 96 sqq.).

24. Les énoncés en tant qu'actes de langage complets seraient selon Dik (à vrai dire, après beaucoup d'autres, dans le sillage de Reichenbach, Vendler, Lyons, etc.) analysables au moyen de prédications enchâssées ; ce qu'on peut résumer ainsi (terminologie en partie personnelle) :

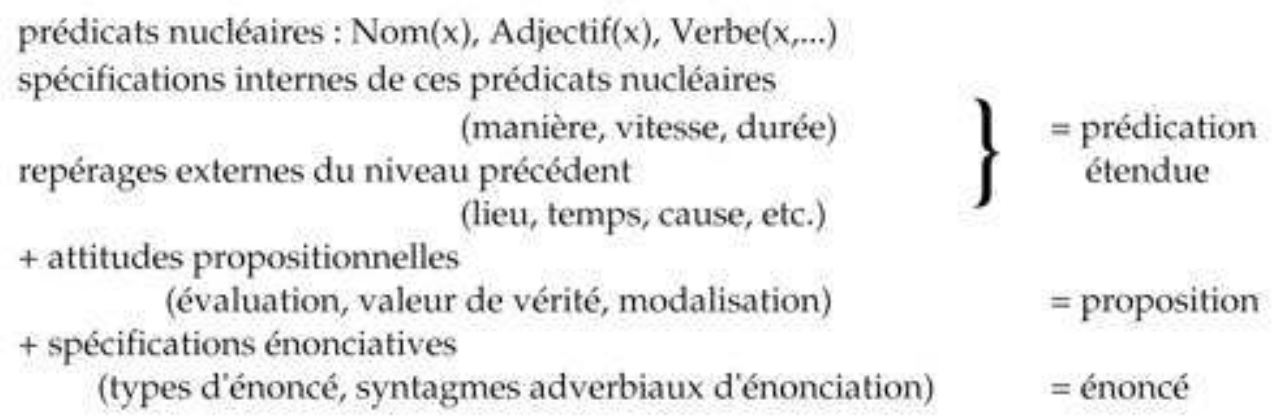




\section{AUTEUR}

\section{ALAIN LEMARÉCHAL}

Université Paris IV - Sorbonne 